\title{
Superpixel Image Segmentation of VISTA Expression in Colorectal Cancer: Implications for Immunotherapy
}

\section{Dongling Wu}

Zucker School of Medicine at Hofstra Northwell Health

\section{Sean Hacking}

Zucker School of Medicine at Hofstra Northwell Health

\section{Taisia Vitkovski}

Zucker School of Medicine at Hofstra Northwell Health

Mansoor Nasim ( $\sim$ MNasim@northwell.edu )

Zucker School of Medicine at Hofstra Northwell Health

\section{Research Article}

Keywords: Colorectal Carcinoma, v-domain immunoglobulin suppressor of T cell activation, VISTA, Immunohistochemistry, Superpixel, Clinical trials

Posted Date: December 14th, 2020

DOl: https://doi.org/10.21203/rs.3.rs-120759/v1

License: (9) This work is licensed under a Creative Commons Attribution 4.0 International License. Read Full License 


\section{Abstract}

Colorectal cancer is an overall bad player and accounts for $9 \%$ of all cancers. Today, advancements in immune checkpoint inhibition has provided therapeutics for many, but not all cancer patients. This issue is in part due to the tumoral microenvironment; which plays a significant role in determining response to immune check point therapeutics. This study serves as the first to evaluate a potent inhibitory checkpoint: V-domain immunoglobulin suppressor of T cell activation (VISTA) and its role in CRC. This was evaluated with both conventional light microscope and superpixel image segmentation. Here we found VISTA expression to be associated with low tumor budding, lower tumor stage, high tumor infiltrating lymphocytes, mature stromal differentiation, BRAF mutation status and better disease-free survival in colorectal cancer. When comparing methodologies; superpixel image segmentation better stratified patients into prognostic groups. Anti-VISTA clinical trials are now open and recruiting for patient enrollment for patients with certain advanced solid tumors. Considering raised VISTA expression is associated with improved survival for patients with colorectal cancer: careful, well-designed and robust clinical trials should be pursued in this cancer subtype.

\section{Introduction}

Colorectal cancer is clinically diverse and has a wide spectrum of possible clinical outcomes. It is notable for being one of the most common inherited cancers, although it also occurs sporadically ${ }^{1,2}$. Many patients present with localized disease; while others present with more advanced anatomic extent of disease. Such patients receive more aggressive treatments, often times with immunotherapies; such as those targeting the Program Death-Ligand 1 (PD-L1) axis. For patients with metastatic colorectal cancer with microsatellite instability: Nivolumab has been found to provide clinical response and disease control in the checkmate 142 trial $^{3}$.

However, the world of immunotherapy is not limited to the PD-L1 axis; the tumor microenvironment fosters a complex ecosystem of immune escape. One such marker is v-domain Ig suppressor of $\mathrm{T}$ cell activation (VISTA). Unlike PD-L1, VISTA is mainly expressed in immune stromal cells. This includes hematopoietic cells and myeloid cells; as well as naïve CD4 + and Foxp3 + regulatory T cells ${ }^{4}$. NK-like Tcells, myeloid-derived suppressor cells (MDSCs), such as: macrophages, granulocytes, dendritic cells and immature myeloid cells: work to regulate the immune microenvironment in cancer ${ }^{5}$.

VISTA is on the cutting edge and as of now; studies have described the suppressive effect of VISTA, with a presumed efficacy for anti-VISTA therapy. However, VISTA is highly controversial: it acts as a ligand on antigen-presenting cells, while also serving as a receptor on T cells ${ }^{6}$. Clinical trials with anti-VISTA are ongoing (Clinicaltrials.gov) and studies correlated with the expression and VISTA as a poor prognostic signature will be important in justifying its use. Apart from obvious differences in survival, evaluating VISTA in the setting of the tumoral microenvironment will also be interesting. Immune markers are often associated with tumor infiltrating lymphocytes and molecular alteration such as microsatellite tumoral instability. 
However, other correlations also exist: with the role of tumor budding and immature stromal differentiation yet to be discovered. In breast cancer, immature stromal differentiation: defined by the presence of myxoid stroma, was found to be inversely associated with PD-L1 expression ${ }^{7}$. In colorectal cancer, stromal differentiation has already been validated as significant prognostic signature by numerous studies ${ }^{8-11}$.

Recent advancements have been made in image analysis and segmentation. Including the use of Qupath as an open-source solution for computational pathology and whole slide image analysis ${ }^{12}$. Qupath offers many applications for image analysis, including superpixel segmentation; which has become a popular approach in computer vision: often used to reduce image complexity for digital analysis ${ }^{13}$. Here we will utilize superpixel image segmentation for the purpose of evaluating VISTA expression.

This study is the first to evaluate VISTA expression in colorectal cancer; therefore, its relationship to the tumoral microenvironment remains largely speculative. The role of VISTA in this cancer subtype is important, especially considering that VISTA may function differently in various solid tumor subtypes. Here we work to further understand the role of VISTA in colorectal cancer: where findings could be used to support rationale for ongoing clinical trials.

\section{Materials And Methods}

\section{Institutional Review Board:}

This study received approval for all experimental protocols by the Institutional Review Board (IRB) of the Human Research Protection Program licensing committee at Northwell Health. All methods were carried out in accordance with all guidelines and regulations. Patient consent was not required by the institutional review board (IRB) committee due to the retrospective nature of the study (Northwell Health IRB number: 18-0128).

\section{Study Design:}

A total of 226 cases of colorectal carcinoma diagnosed in our health system were retrospectively analyzed and cases were collected from 2012-2017. Cases were only selected if they were primary resection specimens, had nil adjuvant therapy and availability of sufficient non-frozen, formalin- fixed, paraffin-embedded tumor material for research purposes. One representative block was selected per case from a single-slide containing the largest portion of tumor. VISTA immunohistochemical (IHC) expression was evaluated on 226 cases from these blocks. Hematoxylin and Eosin stained slides were also evaluated for stromal differentiation, tumor budding and tumor-infiltrating lymphocytes. Further clinicopathological data was collected from the electronic medical records for each case and analysed in relation to VISTA expression. In exploratory analyses: VISTA expression was compared to multiple variables including: cancer-free survival, age, gender, tumor budding score, pathologic stage, tumorinfiltrating lymphocytes (TIL), nodal status, tumor grade, stromal differentiation. Mis-match repair (MMR) status, Ki- ras2 Kirsten rat sarcoma viral oncogene homolog (KRAS) and B-Raf Proto-Oncogene (BRAF). 


\section{Immunohistochemistry:}

All the staining was performed on Formalin-fixed and paraffin-embedded tissue blocks. All staining was performed on the Ventana Benchmark Autostainer (Ventana Medical System, Tucson, Arizona), at the Immunopathology Laboratory of Long Island Jewish Medical Center (Northwell Health System, New Hyde Park, NY). The antibodies are rabbit monoclonal primary antibodies and were pre-diluted. The antibody assays we used for this study are VISTA (ProSci Incorporation) MLH-1 (M1, Ventana), PMS2 (EPR 3947, Ventana), MSH2 (G219-1129, Ventana), and MSH6 (44, Ventana).

\section{Slide Digitalization:}

Histological features in this study were assessed using virtual slides. Slides were scanned on Leica Aperio AT2 (Leica Biosystems, Buffalo Grove, Illinois, USA) whole slide scanner at 20x. Aperio vendor agnostic whole slide image viewer was used for pathologists to assess histology features described in our study.

\section{Manual Analysis of VISTA Expression:}

VISTA expression in immune cells (IC) was scored as the proportion of tumor area occupied by VISTA positive IC of any intensity. Staining was constrained to the tumor microenvironment and to different immune cell types VISTA was expressed by both lymphocytes and myeloid cells (macrophages, dendritic cells, and granulocytes).

\section{Superpixel Analysis of VISTA:}

QuPath is an open source for whole slides image analysis ${ }^{12}$. Studies have shown a high accuracy in QuPath image analysis as descried in Bankhead et al. In clinical practice, an individual pathologist manually interprets staining expression; however, this is prone to observer variation. In our study, we adopted QuPath and chose to utilize superpixel image segmentation to evaluate VISTA expression in addition to manual analysis. IHC slides were scanned using Aperio and QuPath version 0.2.1 Superpixel method is used to quantify the staining. 10x hotspots were identified from the whole slide image by a surgical pathologist. Superpixel automatically groups pixel similarity between different cellular populations as described in Wang et $a^{13}$. Pixel similarity was determined by their red, blue and green values $^{13}$.

Hotspots were used to train the Superpixel method. For this, areas of interest were selected and categorized as "Tumor", "Stroma" and "VISTA". Heatmap was generated after training and the percentage is calculated spontaneous. Once the Superpixel has been optimally trained, all hotspots were calculated under the same Superpixel image segmentation model.

We selected Superpixel method over automatic cell quantification for following reasons first described in Wang et al and also been observed in our study. The RBG values for our hotspots remained stable and once we trained the system, it was able to pick up different RBG values from new cases. Overall, it 
allowed for a faster and stable quantification, with minimal changes in behavior. Traditional cell quantification recognizes cellular expression; however, superpixel segmentation makes calculations based on RBG values, resulting in more subcellar information.

\section{Stromal Differentiation:}

For stromal differentiation, scoring was based on the 3-tier grading system proposed by Ueno et al ${ }^{9}$. (Fig. 1). We analyzed the extramural desmoplastic front at low magnification. We categorized keloid-like collagen as thick hyalinized bundles comprised of hypocellular eosinophilic hyalinized collagen, similar to that of a keloid scar. Myxoid stroma was defined as an amorphous stromal substance made of amphophilic material with a basophilic to grey extracellular matrix. This was usually intermingled with randomly oriented hyalinized collagen. As in Ueno et al stroma grading system, stroma was regarded as immature when fibrotic stroma with myxoid changes was observed. In a tumor with no myxoid stroma, stromal differentiation was categorized as intermediate when keloid-like collagen was intermingled with the mature stroma. Finally, stroma differentiation was regarded as mature when the fibrotic stroma did not contain myxoid stroma or keloid-like collagen and most commonly comprised of fine mature collagen fibers stratified into multiple layers.

\section{Tumor Budding:}

For tumor budding assessment, a detailed search was done for the area having the highest grade of tumour budding. After that, the counting of the buds took place in the hotspot region ( $20 \times$ objective lens). According to ITBCC protocol, the tumor budding is graded using 3-tier grading system as follows: Bd1: 04 buds, Bd2: 5-9 buds and Bd3: 10 or more buds.

\section{Tumor-Infiltrating Lymphocytes:}

TILs were defined as small blue mononuclear cells which infiltrating between tumor cells. Tumors were assessed with a 4-tier scale at the deepest point of the invasive tumor. This was previously validated for the quantification of inflammatory in colorectal cancer by Klintrup et al ${ }^{14}$. A score of 0 denoted nil inflammatory cells, 1 denoted mild patchy increase in mononuclear cells, while 2 and denoted a moderate (bandlike) and florid (cuplike) inflammatory infiltrate respectively. Scores 2 and 3 frequently are accompanied by destruction of cancer cell islands. Scoring was classified as low grade (0-1) and high grade $(2-3)$.

\section{Mis Match Repair Status:}

MMR status was determined based of manual analysis of immunohistochemical protein expression. Cases showing loss of immunohistochemical staining (<1\%) for any of the following stains: MLH1, PMS2, MSH2 and MSH6 was considered MMR deficient.

\section{Next Generation Genomic Sequencing:}

Molecular testing was performed on a subset of patients: 30 (13\%) underwent BRAF testing and 34 (15\%) underwent molecular testing for KRAS. Genomic alterations of BRAF and KRAS were tested by next 
generation genomic sequencing on formalin-fixed, paraffin embedded tissue. Mutational analysis was performed at Genpath laboratories (Elwood Park, NJ).

\section{Statistical Analysis:}

The Kaplan-Meier method was used to evaluate cancer-free survival rate as a function of time. The logrank method was used to compare differences between groups. Pearson's correlation coefficient was utilized for correlation between manual and superpixel analysis. Comparative analysis was performed using the two-tailed Fisher exact test and non-paired $T$ test to examine differences in the clinicopathologic profile. Statistical Analysis was performed using Prism Graphpad version 8.4.2 A pvalue $<0.05$ was considered statistically significant.

\section{Results}

\section{Clinicopathologic and Patient Characteristics:}

This study comprised data from 226 patients with colorectal adenocarcinoma who underwent surgical resection at our health system. Surgeries including: block resection, right hemicolectomy, left hemicolectomy, transverse colectomy, sigmoidoscopy, rectosigmoidectomy and abdominal perineal resection. The mean age for our patient cohort is 67.4 . There was a slight female predominance: $51 \%$ female (115) to $49 \%$ male (111). Tumor budding grades were as follows: Absent or low (113).

Intermediate or high (113) Tumoral stage was as follows: pT1-2 (59), pT3-pT4 (167). For TIL, scoring was as follows: Low (158) and High (68). Lymph node status was as follows: N0 (140), N1 (53), N2 (33). Tumor grade was as follows: Well differentiated (161), Poorly differentiated (65). Stromal differentiation was as follows: 115 mature stroma, and 111 patients with immature. Clinicopathological and patient characteristics can be found in Table 1.

\section{VISTA Expression:}

The overall expression of VISTA on manual analysis was $22 \%$, while 21 cases showed $0 \%$ expression and 6 cases showing more than $90 \%$ expression. The overall expression of VISTA on superpixel was $20 \%$, while 15 cases showed $0 \%$ expression and 3 cases showing more than $90 \%$ expression. Pearson's correlation coefficient demonstrated good correlation between manual and superpixel analysis $(r=0.92)$, shown in Fig. 2. Heatmaps for VISTA expression between manual and superpixel analysis can be seen in Fig. 3.

\section{VISTA Expression and disease-free survival:}

All of the 226 patients are followed with a mean follow up time of 1054 days. Nonlinear regression found the optimal cutoff for VISTA staining and survival to hover at the $20 \%$ expression mark for both manual and superpixel analysis. After setting up the cutoff of high positive value for $20 \%$, we found the correlates of high VISTA expression with favorable survival rates $(P<0.05)$, as shown in Fig. 4. 
For manual analysis: the mean disease-free period for VISTA expression $>20 \%$ is 789 days, 197 days longer than those with VISTA expression $<20 \%$. During the follow-up period, $85 \%$ cases with VISTA expression $>20 \%$ were disease free compare to the $70 \%$ disease free rate for those with VISTA expression $<20 \%$. For superpixel, the mean disease-free period for VISTA expression $>20 \%$ is 787 days, 164 days longer than those with VISTA expression $<20 \%$. During the follow-up period, $90 \%$ cases with VISTA expression $>20 \%$ were disease free compare to the $65 \%$ disease free rate for those with VISTA expression $<20 \%$.

\section{VISTA Expression and the Clinicopathologic Profile:}

For tumor budding, high tumor budding was found to be associated with low VISTA expression $(P=0.01)$ on superpixel analysis. High pathologic tumoral stage was also found associated with low VISTA expression on superpixel analysis $(P=0.04)$. High tumor-Infiltrating lymphocyte scoring was found to correlate with higher VISTA expression on superpixel analysis $(P=0.01)$. When analyzing stroma differentiation, low VISTA expression was found to correlate with immature stroma differentiation by superpixel analysis $(P=0.02)$. Mature stroma had the highest expression, with a mean positivity of $24 \%$, whereas immature stroma had a mean positivity of $17 \%$. Age, gender, lymph node status and tumor grade did not corelate significantly with VISTA expression $(P>0.05)$. Results from Fisher exact analysis and T test can be seen in Table 1 and Fig. 5 . 
Table 1

Fisher Exact Analysis for Clinicopathologic Profile. Significant features $(P \leq 0.05)$ are shown in bold. VISTA, V-domain immunoglobulin suppressor of T cell activation; TBD, Tumor Budding Grade; PT, Stage; N, Nodal Stage; TIL, TumorInfiltrating Lymphocytes; G, Grade.

\begin{tabular}{|c|c|c|c|c|c|c|}
\hline \multirow{2}{*}{$\begin{array}{l}\text { Variable } \\
\text { Cutoff }\end{array}$} & \multicolumn{2}{|c|}{ Manual } & \multirow[t]{2}{*}{ P-Value } & \multicolumn{2}{|c|}{ Superpixel } & \multirow[t]{2}{*}{ P-Value } \\
\hline & $<20 \%$ & $>20 \%$ & & $<20 \%$ & $>20 \%$ & \\
\hline \multicolumn{7}{|l|}{ Age } \\
\hline$<70$ & 72 & 53 & 0.11 & 85 & 40 & 0.21 \\
\hline$\geq 70$ & 47 & 54 & & 60 & 41 & \\
\hline \multicolumn{7}{|l|}{ Gender } \\
\hline Male & 67 & 51 & 0.79 & 78 & 40 & 0.41 \\
\hline Female & 52 & 56 & & 67 & 41 & \\
\hline \multicolumn{7}{|l|}{ Tumor Budding } \\
\hline TBD1 $(<5)$ & 63 & 50 & 0.42 & 63 & 50 & 0.04 \\
\hline TBD2 or BD3 & 56 & 57 & & 82 & 31 & \\
\hline Pathologic Stage & & & 0.03 & & & 0.04 \\
\hline$\leq \mathrm{PT} 2$ & 24 & 35 & & 29 & 30 & \\
\hline РT3-PT4 & 95 & 72 & & 116 & 51 & \\
\hline TIL & & & 0.4 & & & 0.01 \\
\hline Low & 80 & 78 & & 134 & 24 & \\
\hline High & 39 & 29 & & 11 & 57 & \\
\hline Lymph node Stage & & & 0.21 & & & 0.16 \\
\hline NO & 70 & 70 & & 79 & 61 & \\
\hline N1 & 27 & 26 & & 48 & 5 & \\
\hline N2 & 22 & 11 & & 18 & 15 & \\
\hline Tumor grade & & & 0.06 & & & 0.24 \\
\hline G1-G2 & 91 & 70 & & 104 & 57 & \\
\hline G3 & 28 & 37 & & 41 & 24 & \\
\hline Stroma & & & 0.35 & & & 0.02 \\
\hline Mature & 57 & 58 & & 70 & 45 & \\
\hline
\end{tabular}




\begin{tabular}{|lllllll|}
\hline Variable & Manual & P-Value & Superpixel & P-Value \\
\hline Immature & 62 & 49 & 75 & 36 & \\
\hline
\end{tabular}

\section{VISTA Expression and Selected Biomarkers:}

Out of 226 patients, 139 had intact mis match repair, while 47 patients were mis match repair deficient. Molecular testing was performed on a subset of patients: 30 (13\%) had BRAF testing and $34(15 \%)$ had KRAS mutation status. For VISTA expression, we found that Braf mutation status correlated with VISTA expression. Such that high VISTA expression was associated with Braf mutant status $(p<0.002)$ on superpixel analysis. However, MMR and Kras mutation status was not found to be associated with VISTA expression $(P>0.05)$, as shown in Table 2 and Fig. 6.

Table 2

Fisher exact Analysis for Selected Biomarkers. Significant features $(P \leq$ $0.05)$ are shown in bold. VISTA, V-domain immunoglobulin suppressor of $T$ cell activation; PHH3, Phosphohistone H3; MMR, Mismatch repair gene; KRAS, Ki- ras2 Kirsten rat sarcoma viral oncogene homolog BRAF, B-Raf Proto-Oncogene.

\begin{tabular}{|lllllll|}
\hline Variable & Manual & & P-Value & Superpixel & P-Value \\
\hline Cutoff & $<20 \%$ & $>20 \%$ & & $<20 \%$ & $>20 \%$ & \\
MMR Status & & & 0.23 & & & 0.23 \\
\hline Intact & 63 & 76 & & 82 & 57 & \\
Deficient & 16 & 31 & & 23 & 24 & \\
KRAS & & & 0.24 & & & 0.9 \\
Wild-type & 12 & 7 & & 14 & 5 & \\
\hline Mutant & 13 & 2 & & 11 & 4 & \\
\hline BRAF & & & 0.08 & & & 0.002 \\
\hline Wild-type & 10 & 6 & & 14 & 2 & \\
\hline Mutant & 13 & 1 & & 4 & 10 & \\
\hline
\end{tabular}

\section{Discussion}

Overall, immune checkpoint targets have been studied extensively in colorectal cancer. However, VISTA is a relatively novel immune checkpoint and its significance was previously investigated in both esophageal and gastric adenocarcinomas ${ }^{15,16}$. For VISTA and colorectal cancer, our findings also suggest VISTA to be a clinically significant biomarker. 
Firstly, we found VISTA correlates with stromal differentiation, a novel concept described recently that associated with prognosis outcomes in colonic adenocarcinoma ${ }^{8}$. We found higher VISTA expression to correlate with more mature stroma: well depicted by superpixel image segmentation (Fig. 7).

Tumor budding and TILs were also found to be associated with VISTA expression, suggesting that a histologic phenotype exists which support VISTA expression in colorectal cancer. Our study suggests this phenotype includes: high VISTA expression, low tumor budding, mature stromal differentiation, lower tumor stage and longer survival.

Regarding the prognostic findings in our study, it is important to understand that VISTA is a complicated immune check point regulator. One which is present on both CD4 + and Foxp3 + regulatory T cells as well as myeloid-derived suppressor cells MDSCs. In esophageal adenocarcinoma, significant VISTA expression was found on CD $4+T$ cells: which resulted in improved prognostic outcomes ${ }^{15}$. Such prognostic findings were also found in our study.

As a hypothesis, it is possible that survival outcomes in our studies are due to VISTA and its role in converting naïve T cells into FoxP3 expressing T cells, which has been demonstrated before ${ }^{17}$. Importantly, FoxP3 has been shown to improve survival in patients with colorectal cancer 2,18 .

FoxP3 is known to be a transcriptional repressor of the proto-oncogene SKP2, which regulates the cell cycle in the G2/M phase ${ }^{2,19}$. Inhibited FoxP3 expression results in the overexpression of SKP2 and cell proliferation.

Saying this, it is possible that VISTA in this setting may be immune-protective for patients with colorectal cancer. On the other hand, VISTA expression in melanoma was found to be a poor prognostic marker 20 . In essence, this may represent the multifaceted role of VISTA for different tumor subtypes. Clinical trials will have the ultimate say in determining therapeutic efficacy; however, anti-VISTA therapies may not be fruitful in this cancer subtype.

In our study, we utilized Qupath: an open access image analysis software. While doing so, we compared the results with that of standard manual analysis. Overall, superpixel analysis outperformed its manual counterpart. The separation of prognostic cohorts was more statistically significant, and superpixel correlated better with the overall clinicopathologic profile.

In computer vision the use of image segmentation dissociated image segments into colored sets of pixels (super-pixels). This allows for more rudimentary image representations, which allows for ease of analysis. As this sounds ideal for the purpose of biomarker expression in the field of pathology, we decided to super-pixelate VISTA hotspots for the purpose of classification.

There has been some talk of superpixel analysis in the field of medicine. In radiology, brain and breast radiography has been targeted as a novel segmentation method with improvements over current state of the art 21,22 . In the field of histopathology, it has been shown to improve segmentation results for deep 
learning based topologies in cervical intraepithelial neoplasia ${ }^{23}$. In future, superpixel image segmentation could be integrated into the ever-evolving digital pathology ecosystem and becomes ubiquitous to the analysis of biomarker expression. This will facilitate the evolution of the tradition to fully digital pathology workflow.

Regarding the use of hotspots for image analysis in our study; studies have found that hotspot analysis to outperform global scoring. Robertsona et al. found that hotspot scoring had double the potential for predicting recurrence free survival, in the setting of Ki-67 immunohistochemistry and breast cancer ${ }^{24}$. The analysis of hotspots may better correlate with immune activity and future applications in deep learning will allow for automatic detection of hotspots from whole slide images: alleviating the need for manual evaluation 25 .

There are several pitfalls to our study and it is important to consider that this was a retrospective study with the potential for bias. Secondly, we did not perform molecular testing for VISTA expression, which would have been more thorough. Thirdly, we did not perform multiplex immunolabeling in this study, which would have allowed us to identify VISTA expression in different immune cell subtypes. This would have been particularly useful for identifying CD4 + and FoxP3 expressing T cells.

One novel finding is the association for BRAF mutations and higher VISTA expression. This has never been reported; however, a recent publication has shed light onto these phenomena, where BRAF inhibition in BRAF mutant patients increased FOXP3, while downregulating VISTA expression ${ }^{26}$. Our findings support that the BRAF mutant mutation to be associated with higher VISTA expression. While the role of BRAF in the tumoral microenvironment is not well known; it is possible that BRAF may have therapeutic effect through modulation of VISTA expression. The relationship between BRAF and VISTA should be explored in future studies. Today, BRAF inhibitor therapy happens to be ubiquitous to chemotherapy, particularly for patients with melanoma 27.

For VISTA, there are many questions still remain. Future studies examining the therapeutic efficacy of anti-VISTA, in combination with other immune check point inhibitors, will also be useful.

Looking forward, VISTA could be on the cutting edge of an immunotherapy revolution. However, targeting VISTA in an inhibitory fashion should be performed with caution. It may be immune-protective for patients with colorectal cancer.

\section{Declarations}

\section{Contributions}

M.N. arranged all the cases and slides. D.W. and S.H. gathered all the pathology and clinical data. D.W. T.V., M.N. and S.H. graded stroma as well as TIL, T stage, tumor budding. T.V. analyzed IHC staining. D.W. analyzed IHC staining using Superpixel. D.W. and S.H. performed the data analysis. D.W., S.H., and M.N. edited the manuscript. All authors reviewed the manuscript. 


\section{Disclosure of interest}

The authors report no conflict of interest.

\section{References}

[1] Jasperson KW, Tuohy TM, Neklason DW, Burt RW: Hereditary and familial colon cancer. Gastroenterology 2010, 138:2044-58.

[2] Sun X, Feng Z, Wang Y, Qu Y, Gai Y: Expression of Foxp3 and its prognostic significance in colorectal cancer. Int J Immunopathol Pharmacol 2017, 30:201-6.

[3] Overman MJ, McDermott R, Leach JL, Lonardi S, Lenz H-J, Morse MA, Desai J, Hill A, Axelson M, Moss RA, Goldberg MV, Cao ZA, Ledeine J-M, Maglinte GA, Kopetz S, André T: Nivolumab in patients with metastatic DNA mismatch repair-deficient or microsatellite instability-high colorectal cancer (CheckMate 142): an open-label, multicentre, phase 2 study. Lancet Oncol 2017, 18:1182-91.

[4] ElTanbouly MA, Croteau W, Noelle RJ, Lines JL: VISTA: a novel immunotherapy target for normalizing innate and adaptive immunity. Semin Immunol 2019, 42:101308.

[5] Gabrilovich DI, Nagaraj S: Myeloid-derived suppressor cells as regulators of the immune system. Nat Rev Immunol 2009, 9:162-74.

[6] Huang X, Zhang X, Li E, Zhang G, Wang X, Tang T, Bai X, Liang T: VISTA: an immune regulatory protein checking tumor and immune cells in cancer immunotherapy. Journal of Hematology \& Oncology 2020, 13:83.

[7] Zhai Q, Fan J, Lin Q, Liu X, Li J, Hong R, Wang S: Tumor stromal type is associated with stromal PD-L1 expression and predicts outcomes in breast cancer. PLoS One 2019, 14:e0223325-e.

[8] Hacking S, Ebare K, Angert M, Lee L, Vitkovski T, Thomas R, Chavarria H, Jin C, Nasim M: Immature Stroma and Prognostic Profiling in Colorectal Carcinoma: Development and Validation of Novel Classification Systems. Pathology - Research and Practice 2020, 216:152970.

[9] Ueno H, Kanemitsu Y, Sekine S, Ishiguro M, Ito E, Hashiguchi Y, Kondo F, Shimazaki H, Mochizuki S, Kajiwara Y, Shinto E, Yamamoto J: Desmoplastic Pattern at the Tumor Front Defines Poor-prognosis Subtypes of Colorectal Cancer. Am J Surg Pathol 2017, 41:1506-12.

[10] Ueno H, Shinto E, Kajiwara Y, Fukazawa S, Shimazaki H, Yamamoto J, Hase K: Prognostic impact of histological categorisation of epithelial-mesenchymal transition in colorectal cancer. British Journal of Cancer 2014, 111:2082-90.

[11] Ueno H, Jones AM, Wilkinson KH, Jass JR, Talbot IC: Histological categorisation of fibrotic cancer stroma in advanced rectal cancer. Gut 2004, 53:581. 
[12] Bankhead P, Loughrey MB, Fernández JA, Dombrowski Y, McArt DG, Dunne PD, McQuaid S, Gray RT, Murray LJ, Coleman HG, James JA, Salto-Tellez M, Hamilton PW: QuPath: Open source software for digital pathology image analysis. Scientific Reports 2017, 7:16878.

[13] Wang M, Liu X, Gao Y, Ma X, Soomro NQ: Superpixel segmentation: A benchmark. Signal Processing: Image Communication 2017, 56:28-39.

[14] Klintrup K, Mäkinen JM, Kauppila S, Väre PO, Melkko J, Tuominen H, Tuppurainen K, Mäkelä J, Karttunen TJ, Mäkinen MJ: Inflammation and prognosis in colorectal cancer. Eur J Cancer 2005, 41:264554.

[15] Loeser H, Kraemer M, Gebauer F, Bruns C, Schröder W, Zander T, Persa OD, Alakus H, Hoelscher A, Buettner R, Lohneis $\mathrm{P}$, Quaas A: The expression of the immune checkpoint regulator VISTA correlates with improved overall survival in pT1/2 tumor stages in esophageal adenocarcinoma. Oncoimmunology 2019, 8:e1581546.

[16] Böger C, Behrens H-M, Krüger S, Röcken C: The novel negative checkpoint regulator VISTA is expressed in gastric carcinoma and associated with PD-L1/PD-1: A future perspective for a combined gastric cancer therapy? Oncoimmunology 2017, 6:e1293215-e.

[17] Lines JL, Pantazi E, Mak J, Sempere LF, Wang L, O'Connell S, Ceeraz S, Suriawinata AA, Yan S, Ernstoff MS, Noelle R: VISTA is an immune checkpoint molecule for human T cells. Cancer Res 2014, 74:1924-32.

[18] Kim M, Grimmig T, Grimm M, Lazariotou M, Meier E, Rosenwald A, Tsaur I, Blaheta R, Heemann U, Germer CT, Waaga-Gasser AM, Gasser M: Expression of Foxp3 in colorectal cancer but not in Treg cells correlates with disease progression in patients with colorectal cancer. PLoS One 2013, 8:e53630.

[19] Pagano M: Control of DNA synthesis and mitosis by the Skp2-p27-Cdk1/2 axis. Mol Cell 2004, 14:414-6.

[20] Choi JW, Kim YJ, Yun KA, Won CH, Lee MW, Choi JH, Chang SE, Lee WJ: The prognostic significance of VISTA and CD33-positive myeloid cells in cutaneous melanoma and their relationship with PD-1 expression. Scientific Reports 2020, 10:14372.

[21] Huang Q, Huang Y, Luo Y, Yuan F, Li X: Segmentation of breast ultrasound image with semantic classification of superpixels. Medical Image Analysis 2020, 61:101657.

[22] Verma N, Cowperthwaite MC, Markey MK: Superpixels in brain MR image analysis. $201335^{\text {th }}$ Annual International Conference of the IEEE Engineering in Medicine and Biology Society (EMBC), 2013. pp. 1077-80. 
[23] Sornapudi S, Stanley RJ, Stoecker WV, Almubarak H, Long R, Antani S, Thoma G, Zuna R, Frazier SR: Deep Learning Nuclei Detection in Digitized Histology Images by Superpixels. J Pathol Inform 2018, 9:5-.

[24] Robertson S, Acs B, Lippert M, Hartman J: Prognostic potential of automated Ki67 evaluation in breast cancer: different hot spot definitions versus true global score. Breast Cancer Research and Treatment 2020, 183:161-75.

[25] Saha M, Chakraborty C, Arun I, Ahmed R, Chatterjee S: An Advanced Deep Learning Approach for Ki67 Stained Hotspot Detection and Proliferation Rate Scoring for Prognostic Evaluation of Breast Cancer. Scientific reports 2017, 7:3213-

[26] Rosenbaum S, Knecht M, Mollaee M, Zhong Z, Erkes D, McCue P, Chervoneva I, Berger A, Lo J, Fisher D, Gershenwald J, Davies M, Purwin T, Aplin A: FOXD3 Regulates VISTA Expression in Melanoma. Cell Reports 2020, 30:510-24.e6.

[27] Flaherty KT, Infante JR, Daud A, Gonzalez R, Kefford RF, Sosman J, Hamid O, Schuchter L, Cebon J, Ibrahim N, Kudchadkar R, Burris HA, Falchook G, Algazi A, Lewis K, Long GV, Puzanov I, Lebowitz P, Singh A, Little S, Sun P, Allred A, Ouellet D, Kim KB, Patel K, Weber J: Combined BRAF and MEK Inhibition in Melanoma with BRAF V600 Mutations. New England Journal of Medicine 2012, 367:1694-703.

\section{Figures}


$10 \%$ Superpixel
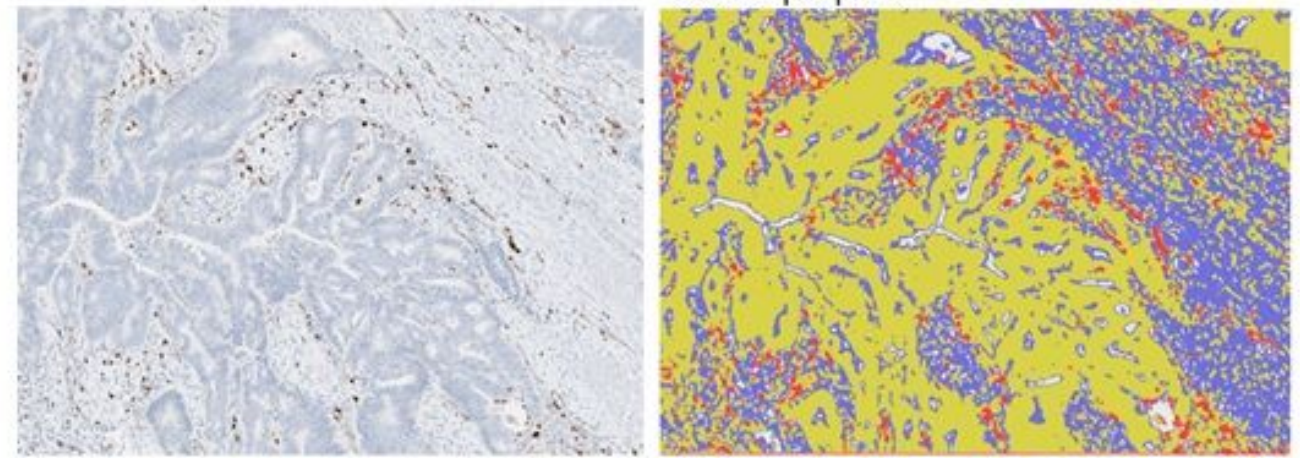

$15 \%$ Superpixel
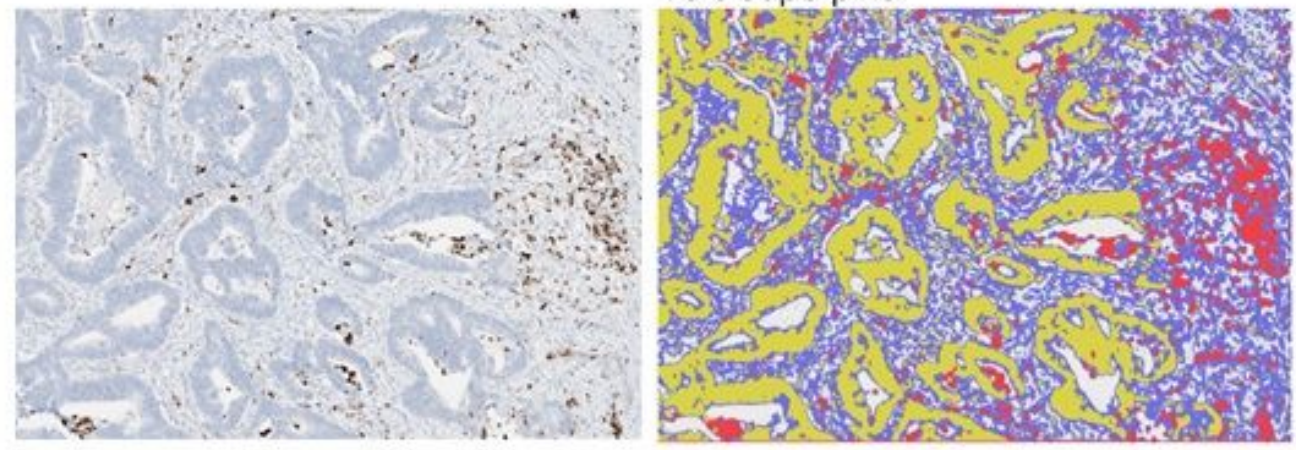

$25 \%$ Superpixel
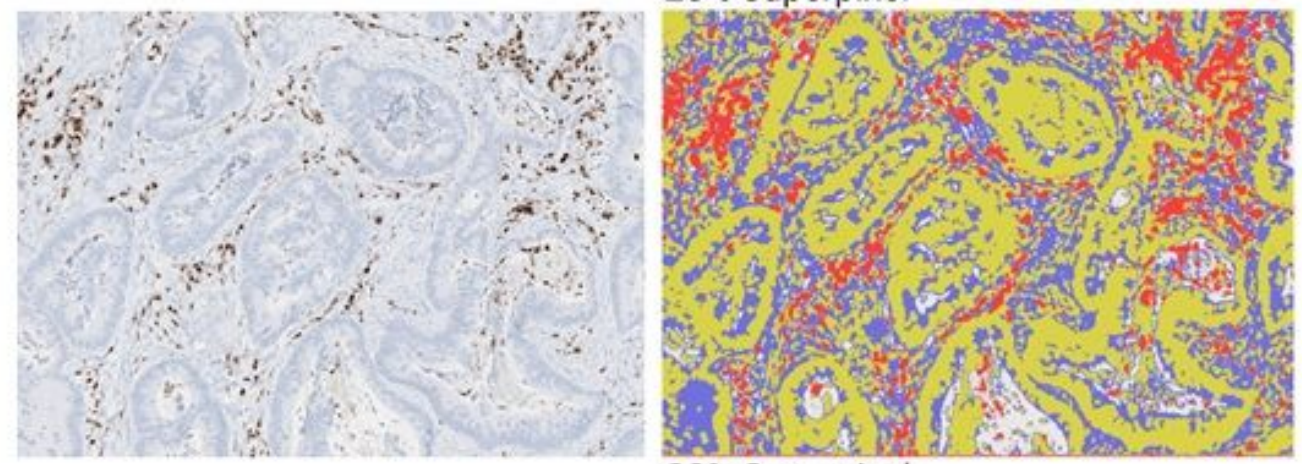

$30 \%$ Superpixel
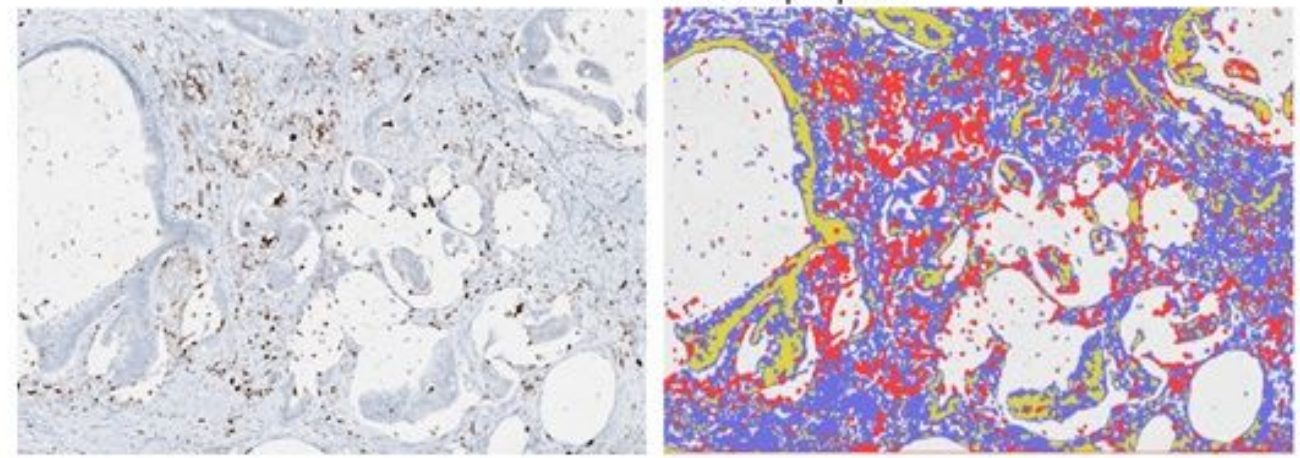

Figure 1

Superpixel Analysis of VISTA Expression. Red, VISTA; Yellow, Tumor; Blue. Stroma 
$10 \%$ Superpixel
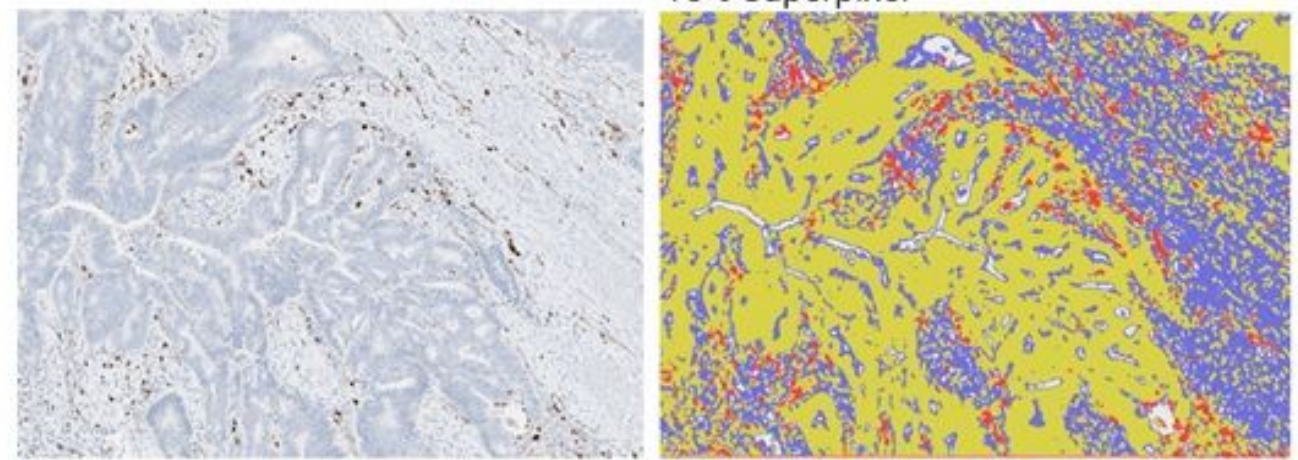

$15 \%$ Superpixel
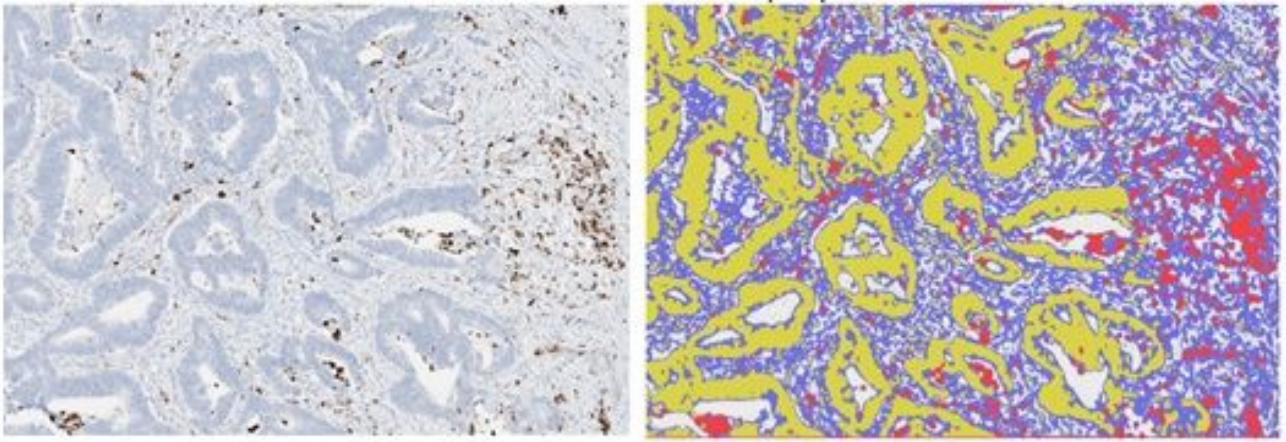

$25 \%$ Superpixel
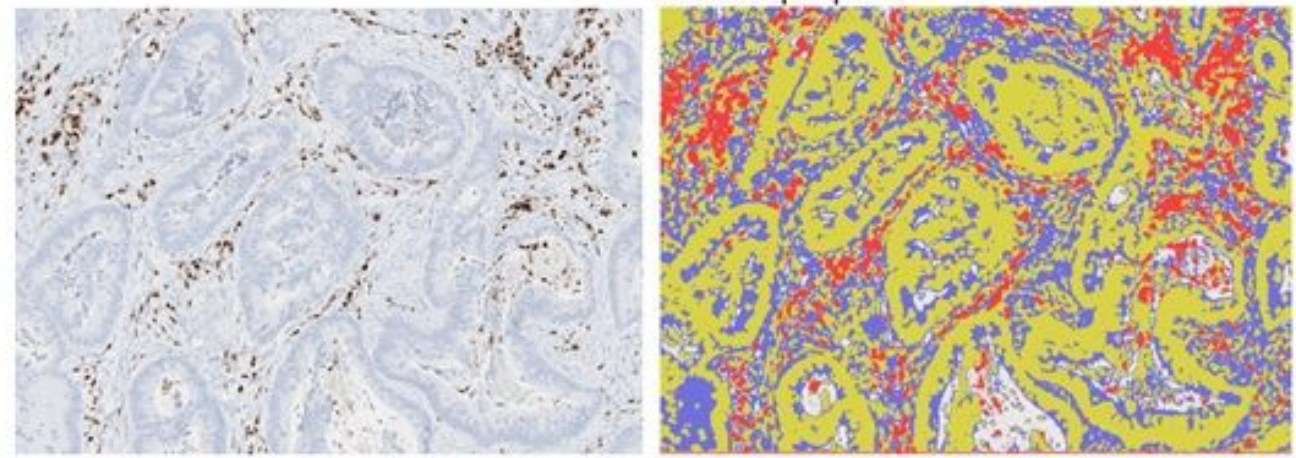

\section{$30 \%$ Superpixel}
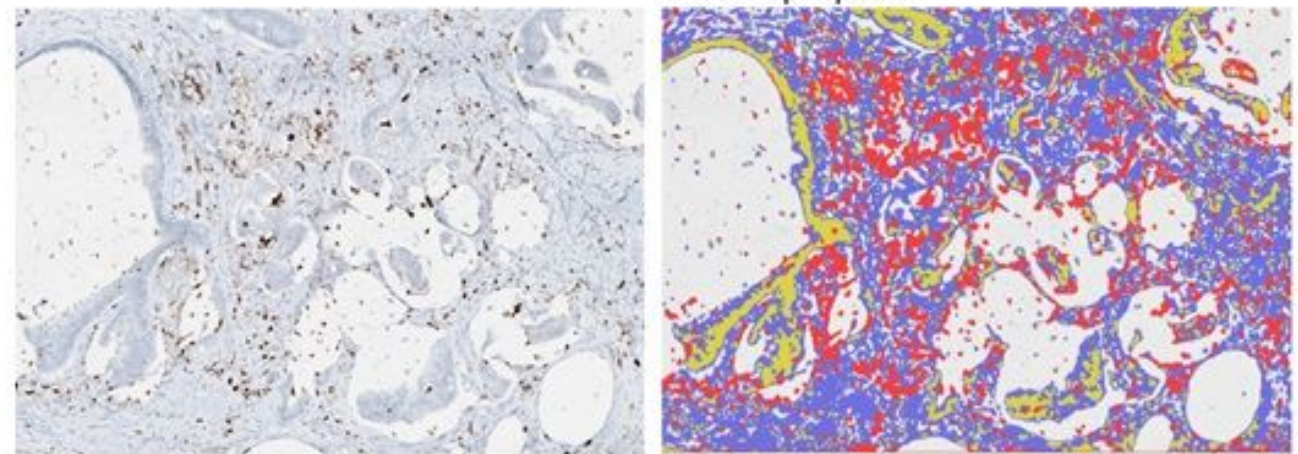

Figure 1

Superpixel Analysis of VISTA Expression. Red, VISTA; Yellow, Tumor; Blue. Stroma 


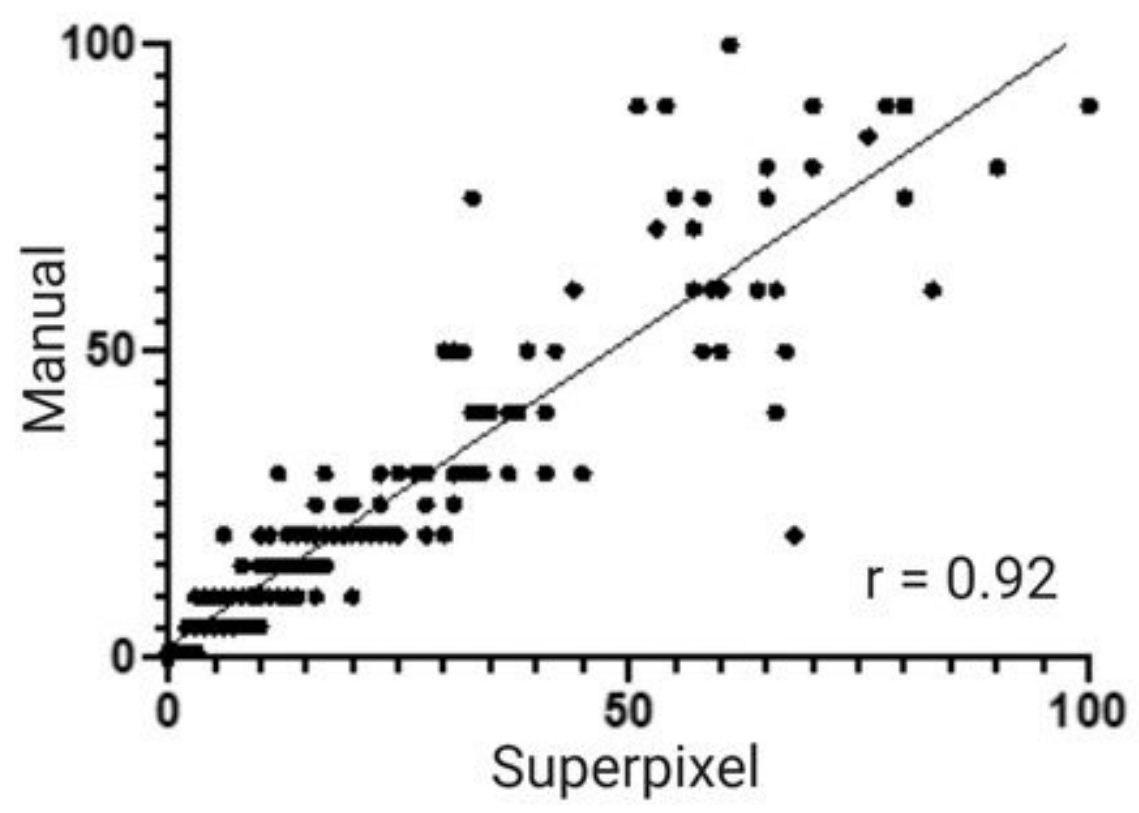

Figure 2

Pearson's Correlation Between Manual and Superpixel Analysis

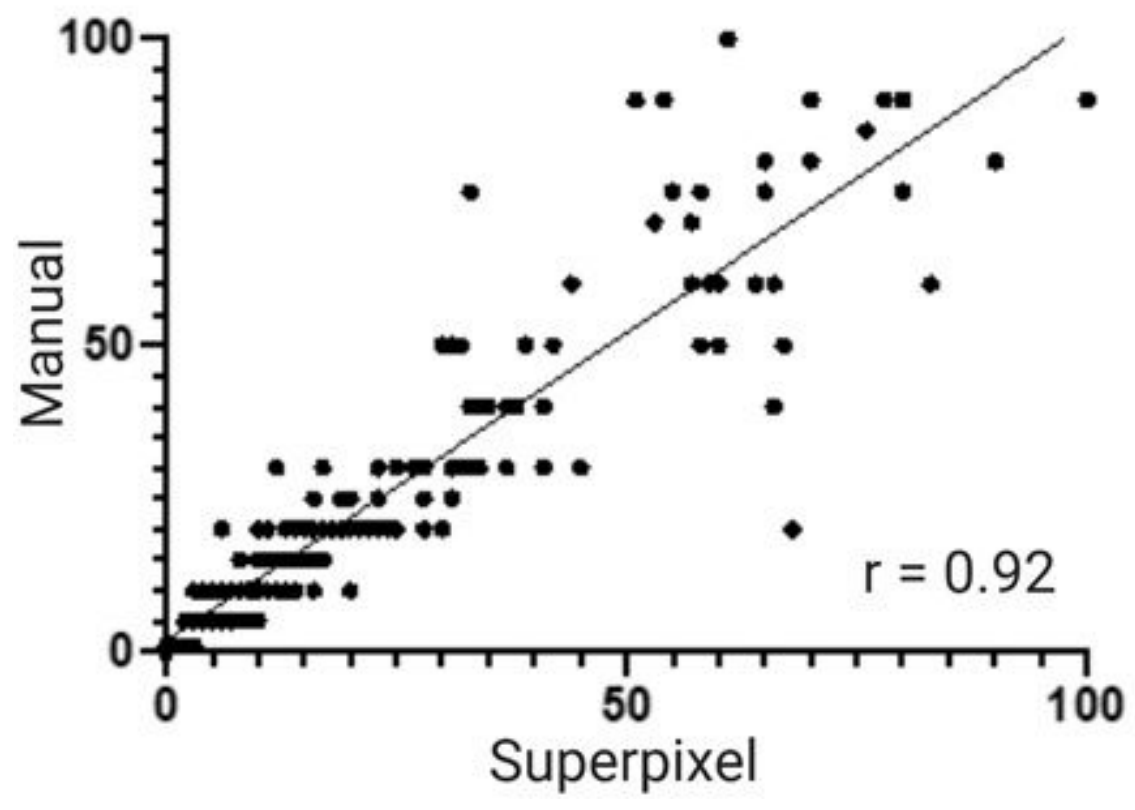

Figure 2

Pearson's Correlation Between Manual and Superpixel Analysis 


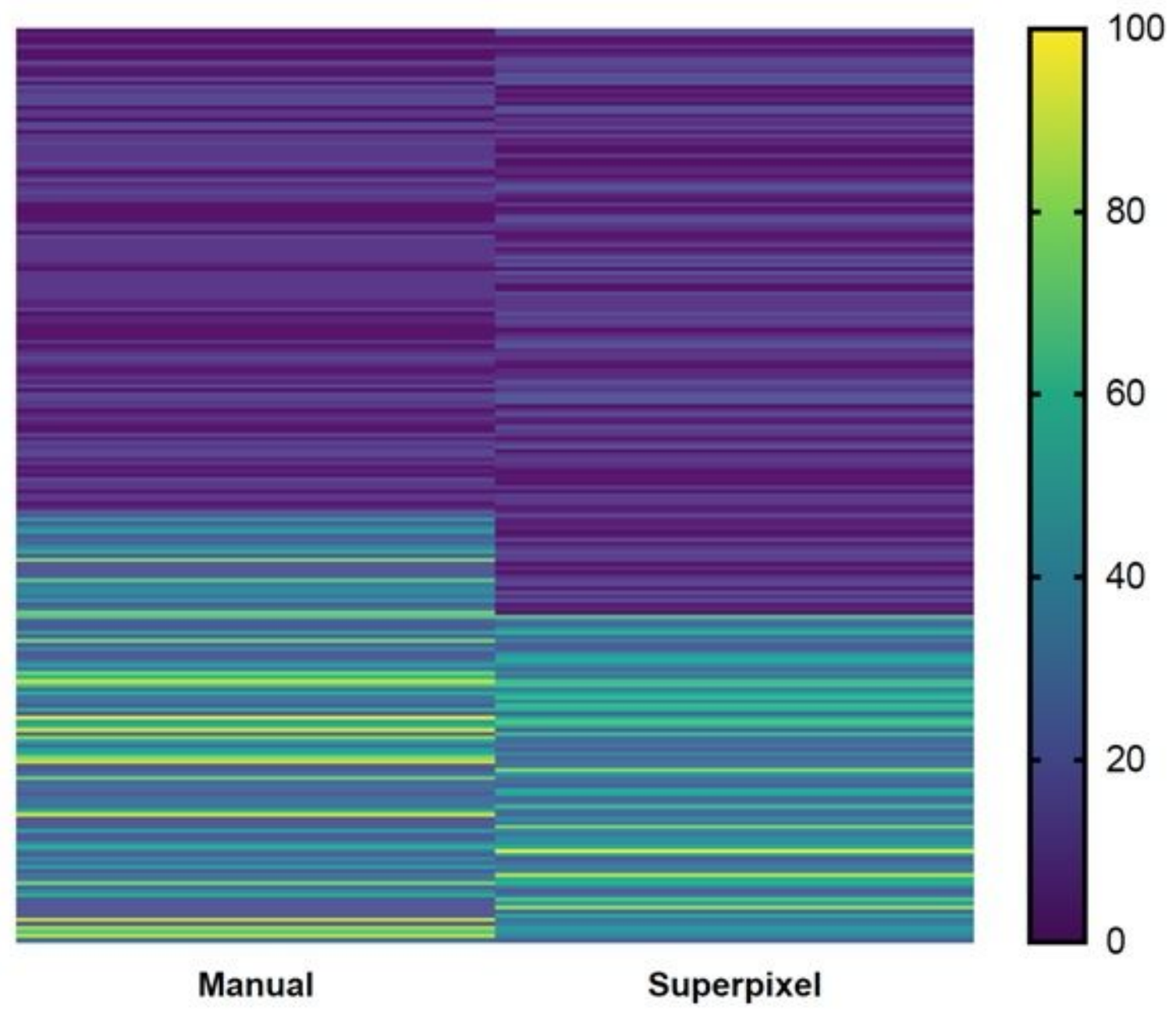

Figure 3

Heatmap for VISTA expression Between Manual and Superpixel Analysis 


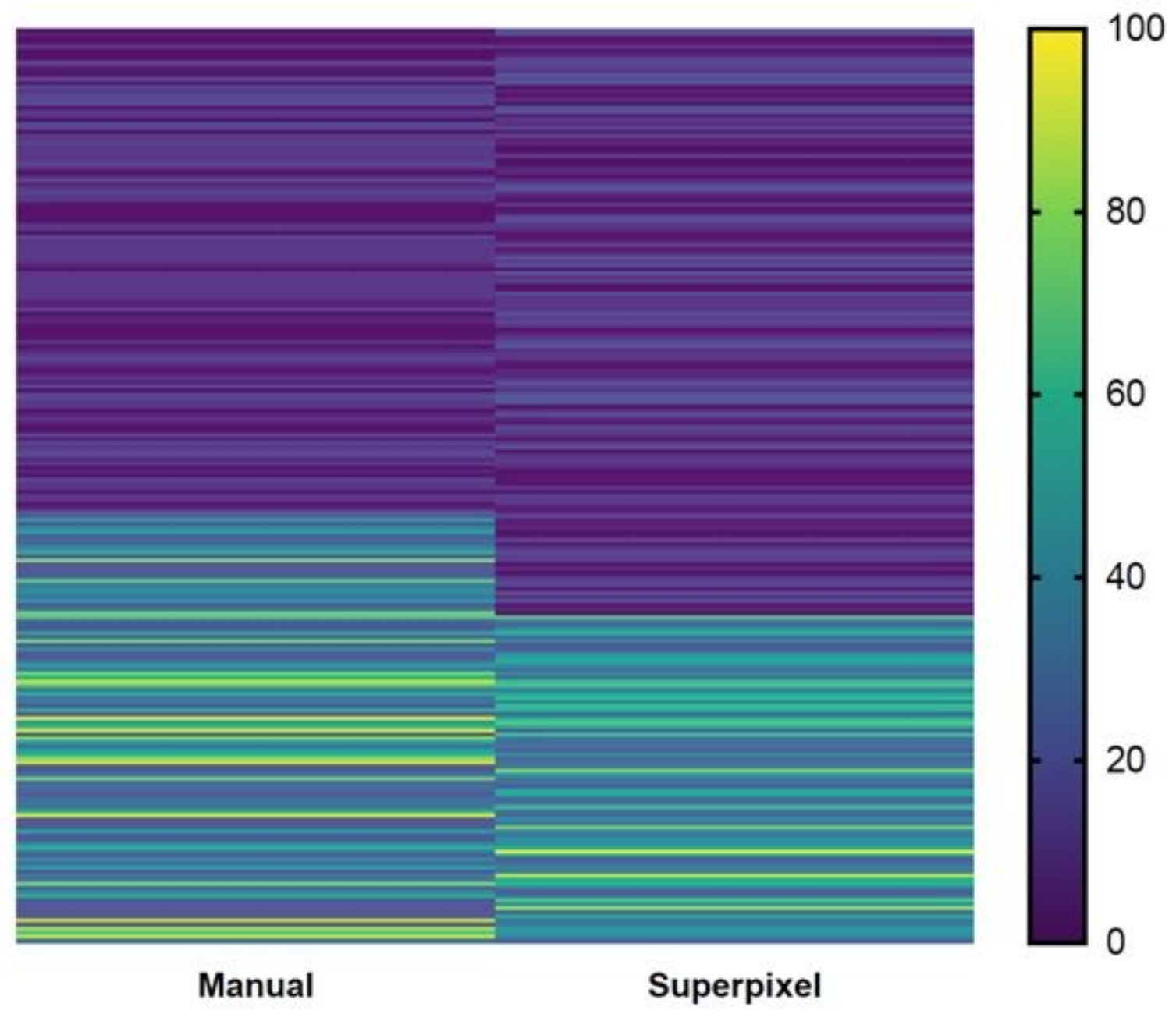

Figure 3

Heatmap for VISTA expression Between Manual and Superpixel Analysis 
Kaplan Meier Survival

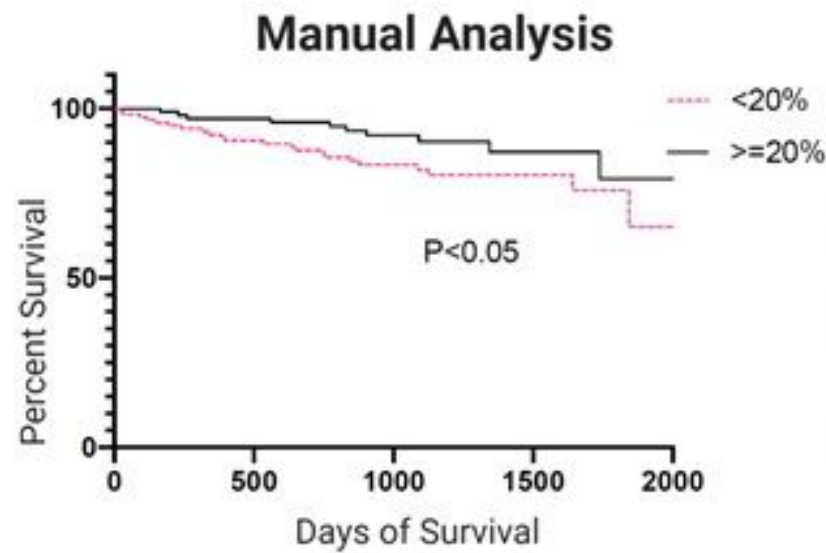

Superpixel Analysis

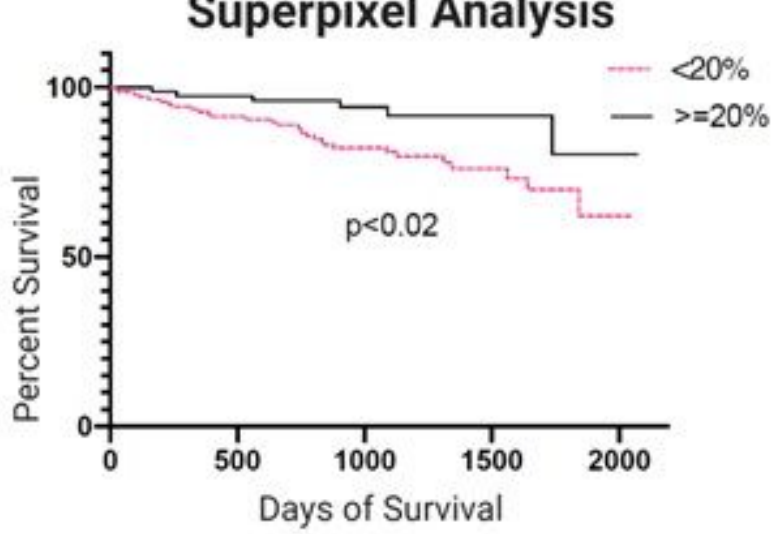

Nonlinear Regression
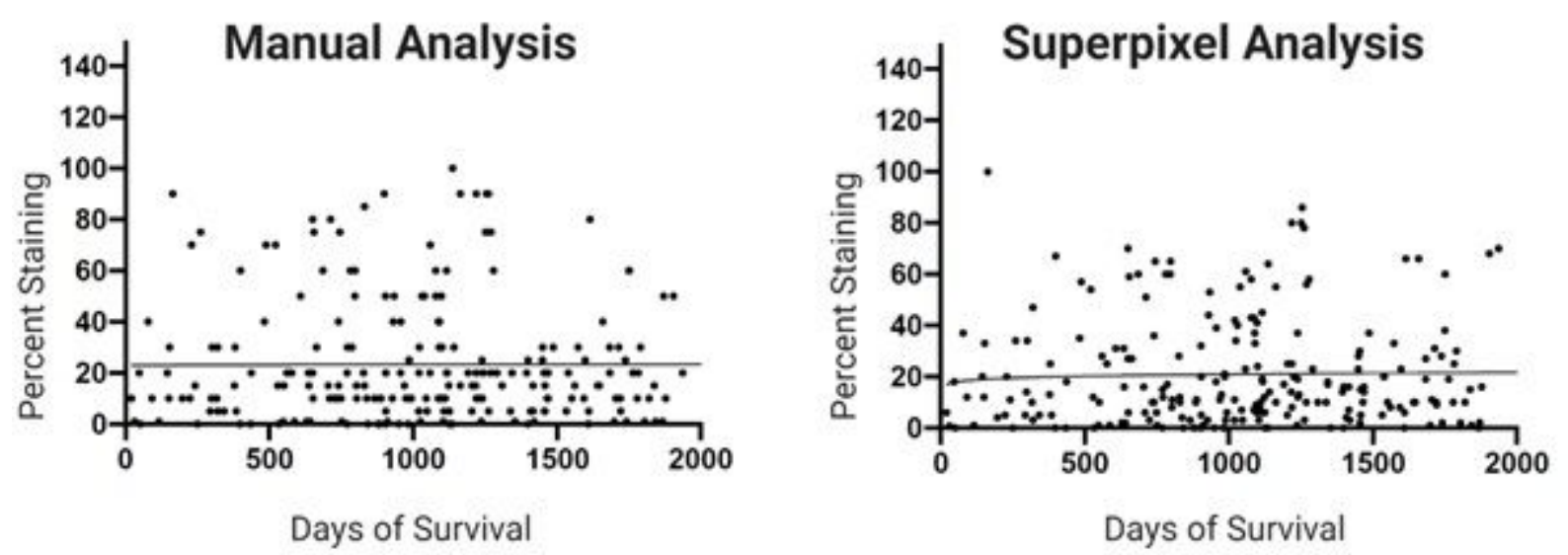

Figure 4

Kaplan Meier Survival and Nonlinear Regression for VISTA Expression: Manual and Superpixel Analysis 


\section{Kaplan Meier Survival}

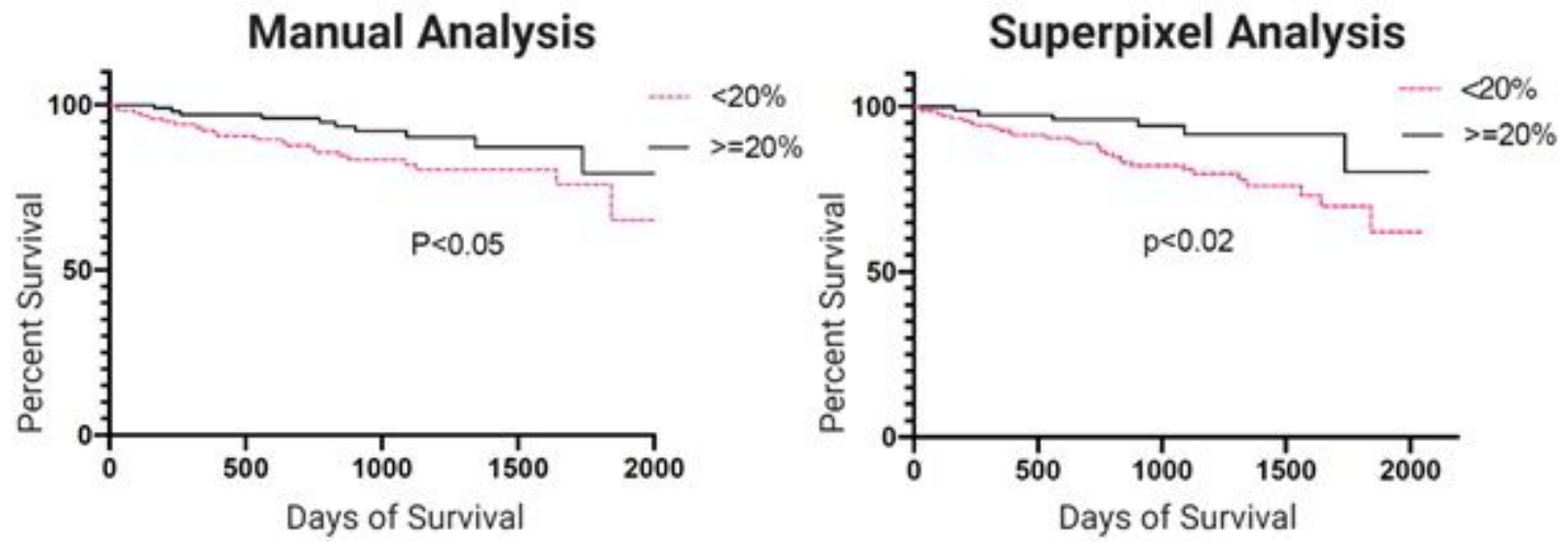

Superpixel Analysis

Nonlinear Regression
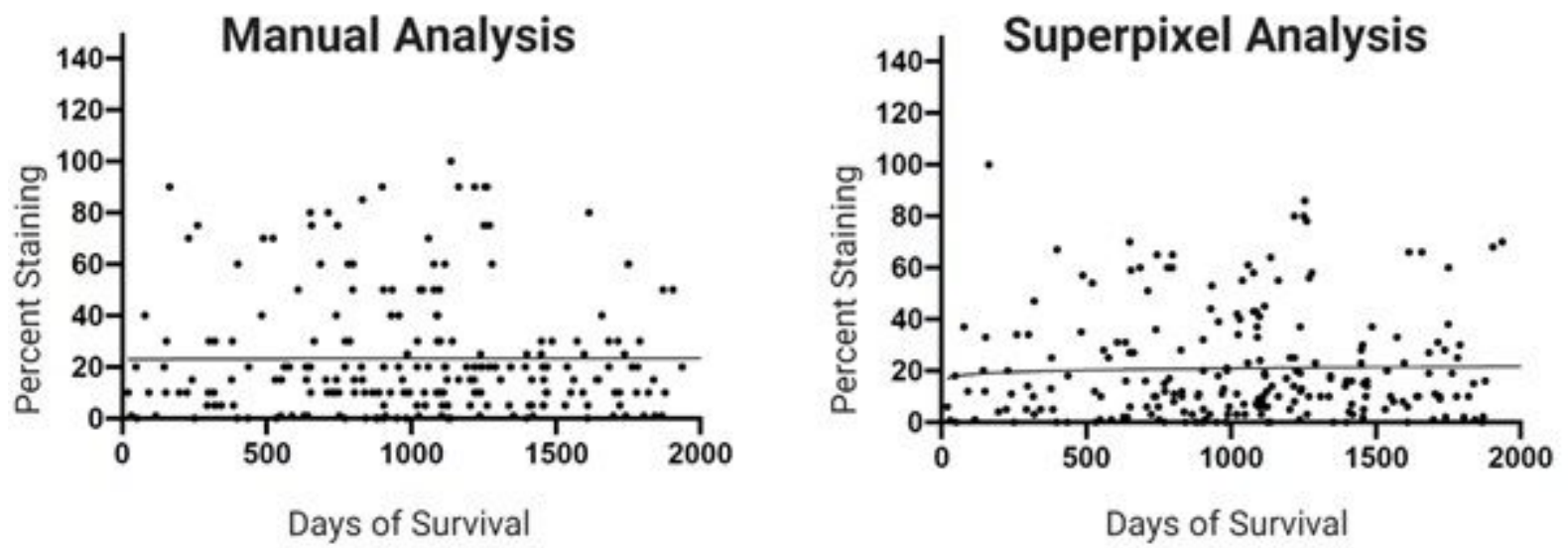

Figure 4

Kaplan Meier Survival and Nonlinear Regression for VISTA Expression: Manual and Superpixel Analysis
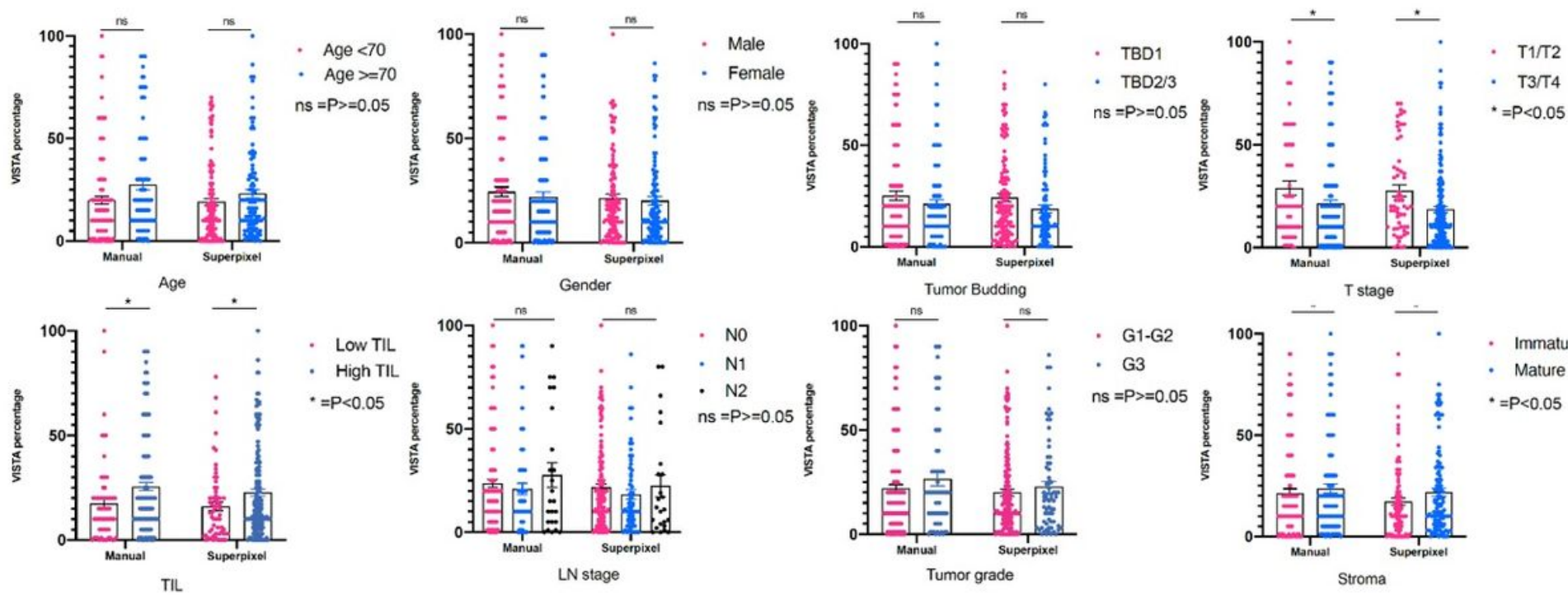

TIL

Figure 5 
T-test for Clinicopathologic Profile. VISTA, V-domain immunoglobulin suppressor of T cell activation; TBD, Tumor Budding Grade; PT, Stage; TIL, Tumor-Infiltrating Lymphocytes; N, Nodal Stage; G, Grade.

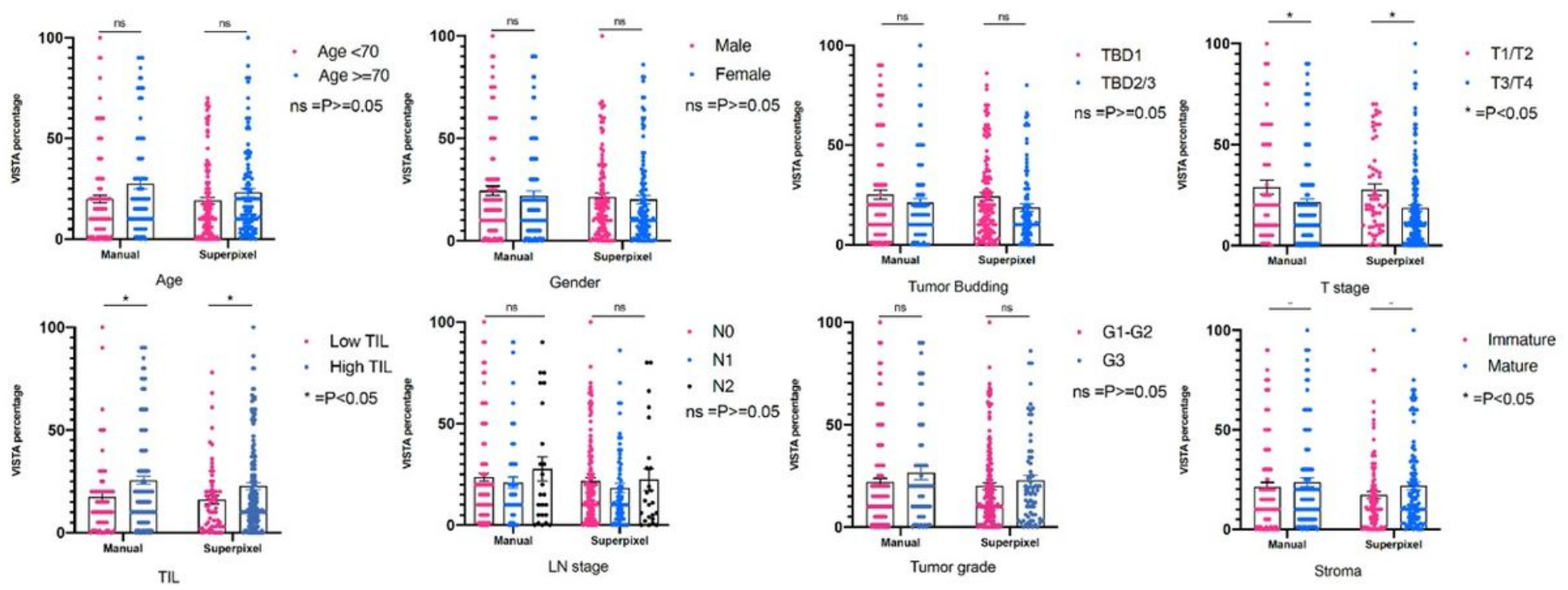

Figure 5

T-test for Clinicopathologic Profile. VISTA, V-domain immunoglobulin suppressor of T cell activation; TBD, Tumor Budding Grade; PT, Stage; TIL, Tumor-Infiltrating Lymphocytes; N, Nodal Stage; G, Grade.
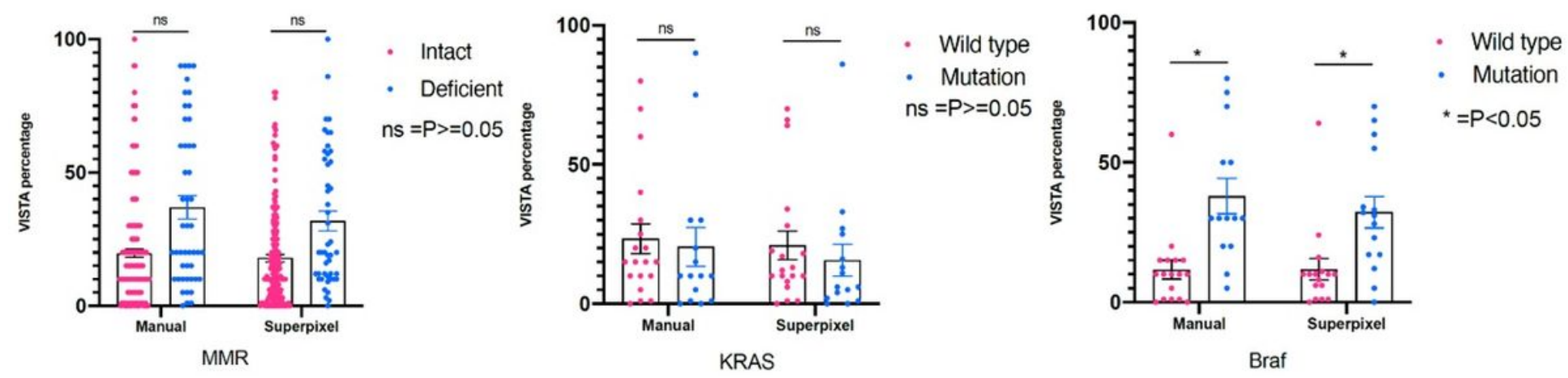

Figure 6

T-test for Selected Biomarkers. VISTA, V-domain immunoglobulin suppressor of T cell activation. MMR, Mismatch repair gene; KRAS, Ki- ras2 Kirsten rat sarcoma viral oncogene homolog BRAF, B-Raf ProtoOncogene. 


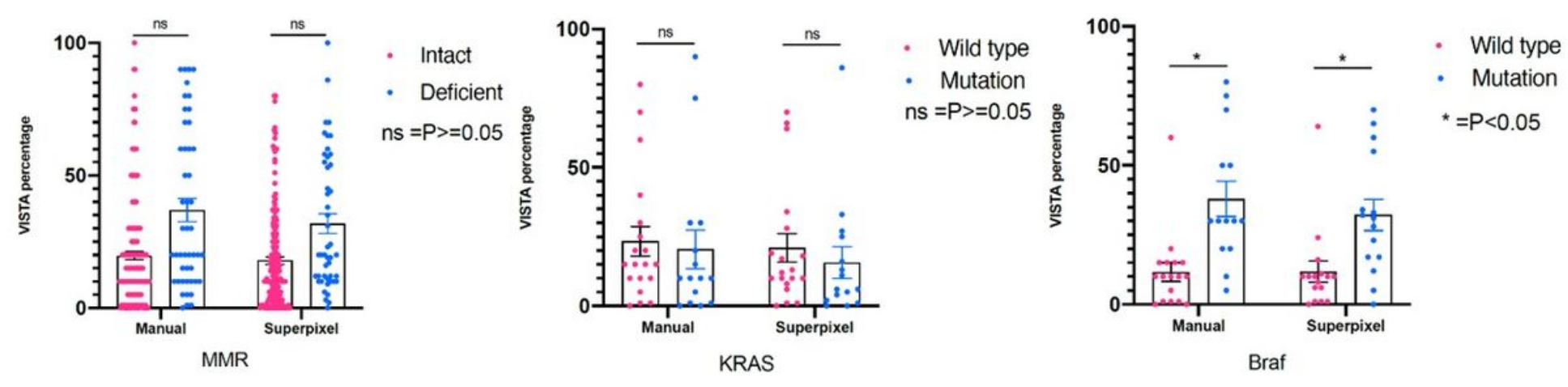

Figure 6

T-test for Selected Biomarkers. VISTA, V-domain immunoglobulin suppressor of T cell activation. MMR, Mismatch repair gene; KRAS, Ki- ras2 Kirsten rat sarcoma viral oncogene homolog BRAF, B-Raf ProtoOncogene.
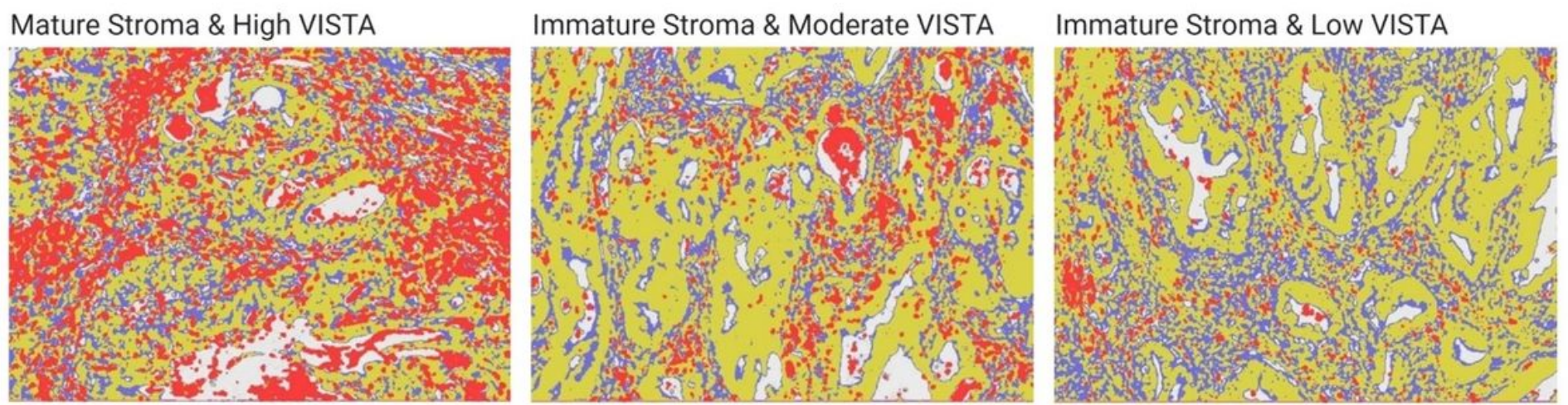

$\square$ VISTA

STROMA

TUMOR

Figure 7

Decreased VISTA Expression and Immature Stromal Differentiation. VISTA, V-domain immunoglobulin suppressor of T cell activation.
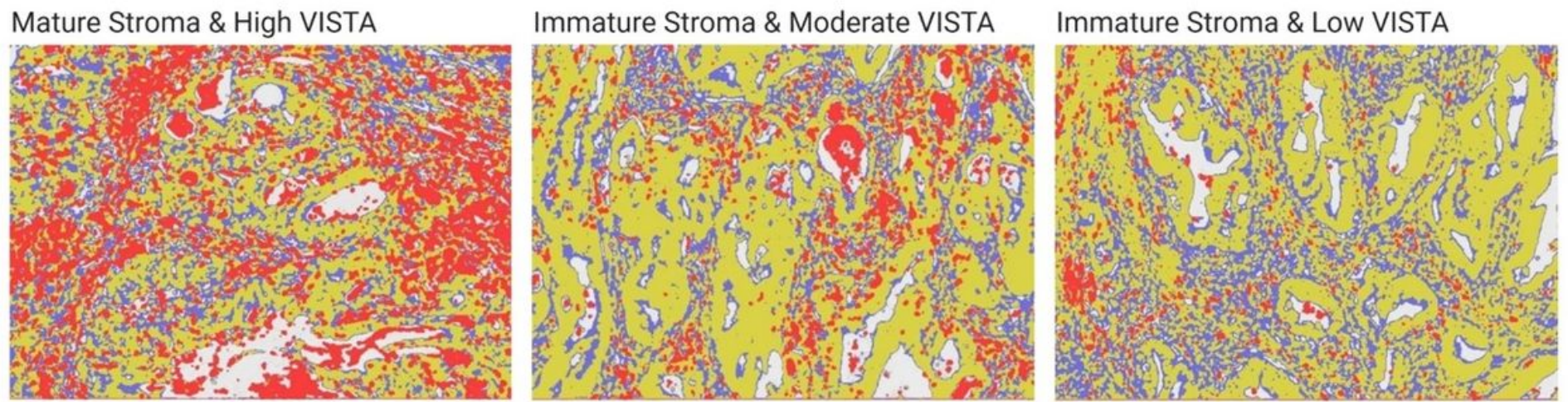

$\square$ VISTA

STROMA

TUMOR

\section{Figure 7}


Decreased VISTA Expression and Immature Stromal Differentiation. VISTA, V-domain immunoglobulin suppressor of T cell activation. 